\title{
Morphology-Dependent Resonances of an Infinitely Long Circular Cylinder Illuminated by a Diagonally Incident Plane Wave or a Focused Gaussian Beam
}

James A. Lock

Cleveland State University, j.lock@csuohio.edu

Follow this and additional works at: https://engagedscholarship.csuohio.edu/sciphysics_facpub

Part of the Physics Commons

How does access to this work benefit you? Let us know!

\section{Publisher's Statement}

This paper was published in Journal of the Optical Society of America A: Optics Image Science and Vision and is made available as an electronic reprint with the permission of OSA. The paper can be found at the following URL on the OSA website: http://www.opticsinfobase.org/josaa/ abstract.cfm?URI=josaa-14-3-653. Systematic or multiple reproduction or distribution to multiple locations via electronic or other means is prohibited and is subject to penalties under law.

\section{Original Citation}

Lock, James A. "Morphology-Dependent Resonances of an Infinitely Long Circular Cylinder Illuminated by a Diagonally Incident Plane Wave or a Focused Gaussian Beam." Journal of the Optical Society of America A: Optics Image Science and Vision 14 (1997): 653-661.

\section{Repository Citation}

Lock, James A., "Morphology-Dependent Resonances of an Infinitely Long Circular Cylinder Illuminated by a Diagonally Incident Plane Wave or a Focused Gaussian Beam" (1997). Physics Faculty Publications. 70.

https://engagedscholarship.csuohio.edu/sciphysics_facpub/70

This Article is brought to you for free and open access by the Physics Department at EngagedScholarship@CSU. It has been accepted for inclusion in Physics Faculty Publications by an authorized administrator of EngagedScholarship@CSU. For more information, please contact library.es@csuohio.edu. 


\title{
Morphology-dependent resonances of an infinitely long circular cylinder illuminated by a diagonally incident plane wave or a focused Gaussian beam
}

\author{
James A. Lock \\ Department of Physics, Cleveland State University, Cleveland, Ohio 44115
}

Received April 11, 1996; revised manuscript received September 23, 1996; accepted October 10, 1996

The $S$ matrix for scattering a diagonally incident focused Gaussian beam by an infinitely long circular cylinder is calculated, and the diagonal and off-diagonal elements of the matrix are physically interpreted. In addition, the TE and TM morphology-dependent resonances of the cylinder for plane-wave incidence are examined, and it is found that the cylinder size parameter at resonance increases as the angle of incidence of the plane wave increases. The size parameter increase is explained in terms of the heuristic model of morphologydependent resonances. The presence of both TE and TM resonances in each of the $\epsilon$-polarized and $\mu$-polarized partial-wave scattering amplitudes is discussed. This mixing is interpreted in terms of successive polarization-preserving and cross-polarized total internal reflections of the morphology-dependent-resonance wave inside the cylinder. Finally, some novel properties of morphology-dependent resonances produced by Gaussian-beam incidence are predicted. (C) 1997 Optical Society of America [S0740-3232(97)00803-X]

\section{INTRODUCTION}

In electromagnetic scattering by an object with a high degree of symmetry, such as a sphere, a spheroid, or a cylinder, morphology-dependent resonances (MDR's) occur for various combinations of particle size, refractive index, and wavelength of the incident light. ${ }^{1}$ Mathematically, a MDR occurs when a partial-wave scattering amplitude reaches its maximum value. This is accompanied by the corresponding partial-wave interior amplitude growing to several orders of magnitude above its nonresonant value. Physically, the heuristic model of MDR's describes a resonance as follows. ${ }^{1}$ The ray associated with a given partial wave is incident on the particle just beyond its edge and tunnels through the particle's centrifugal barrier. Once inside the particle it successively internally reflects off the particle surface at an angle past that for total internal reflection. It circumnavigates the particle just inside its surface with constructive interference, thus building up large interior fields. At each internal reflection a small fraction of the light tunnels outward through the centrifugal barrier and escapes to the far zone. This is the light resonantly scattered by the particle.

Various studies have examined the influence on MDR's of (1) the shape and the positioning of the incident beam ${ }^{2}$ and (2) perturbations to the shape of the target particle. 1,3,4 The effect of perturbations of particle geometry is illustrated by the case of a plane wave impinging on a spheroid with end-on incidence, where the spheroid size parameter at resonance is found to be a function of the spheroid eccentricity. The effect of the beam shape and positioning is illustrated by the case of a focused Gaussian beam incident on a sphere, where the resonant size parameters are identical to those occurring for scat- tering of a plane wave. But the positioning of the Gaussian beam dramatically raises or lowers the MDR excitation rate.

In this paper we examine the properties of MDR's produced by either a plane wave or a focused Gaussian beam diagonally incident on an infinitely long homogeneous dielectric circular cylinder. As is the case for plane-wavespheroid scattering, we find that the cylinder resonant size parameters depend on the details of the beamcylinder geometry. As is the case for Gaussian-beamsphere scattering, the positioning of the incident beam affects the MDR excitation rate. But, contrary to Gaussian-beam-sphere scattering, the shape of the incident beam also influences the nature of the MDR's.

The body of this paper proceeds as follows. In a companion paper ${ }^{5}$ that is hereafter denoted as Paper I, I derived the far-zone scattered fields resulting from the interaction of a focused Gaussian beam diagonally incident on an infinitely long homogeneous circular cylinder. Section 2 briefly summarizes the formulas from Paper I that are pertinent to the discussion of MDR's. In Section 3 we consider the diagonally incident beam-cylinder system as a prototype example of a projectile-plus-target system in which a number of different scattering reactions can occur. We derive the interaction $S$ matrix for arbitrary beam-cylinder scattering and demonstrate that it is unitary. In Section 4 we examine the MDR's produced by a diagonally incident plane wave and relate them to the phase of the elements of the $S$ matrix. We find that the cylinder resonant size parameters depend on the angle of incidence of the plane wave and that both TE and TM resonances occur in each scattering channel. In Section 5 the MDR's produced by an incident Gaussian beam are 
discussed, and I comment on the ways in which they differ from the MDR's produced by a plane wave. Finally, in Section 6 the main results are summarized.

\section{PARTIAL-WAVE SCATTERING AMPLITUDES}

The partial-wave scattering and interior amplitudes and the far-zone electric fields and intensity for scattering of a diagonally incident focused Gaussian beam by an infinitely long homogeneous circular cylinder were derived in Paper I. With use of the notation of Paper I the cylinder has radius $a$ and refractive index $n$ and is oriented so that its symmetry axis coincides with the $z$ axis of a rectangular coordinate system. The incident monochromatic Gaussian beam has wave number $k$ and time dependence $\exp (-i \omega t)$. Its dominant propagation direction is in the $x-z$ plane and makes an angle $\xi$ with the $x$ axis. The electric field half-width in the beam's focal plane is $w_{0}$, and the center of its focal waist is at $\left(x_{0}, y_{0}, z_{0}\right)$. This geometry is illustrated in Fig. 1.

The beam, the scattered fields, and the interior fields are $\epsilon$ polarized if their electric fields lie in the horizontal $x-y$ plane, and they are $\mu$ polarized if their magnetic fields lie in the horizontal plane. When an $\epsilon$-polarized incident Gaussian beam is modeled as an angular spectrum of plane waves, the coefficients in its partial-wave expansion are approximately

$$
\begin{aligned}
A_{l}(h)= & \frac{\cos \xi}{2 s \sqrt{\pi}\left(1-h^{2}\right) \sqrt{F}} \\
& \times \exp \left\{i k\left[h z_{0}+\left(1-h^{2}\right)^{1 / 2} x_{0}\right]\right\} \\
& \times \exp \left\{-\left[h \cos \xi+\left(1-h^{2}\right)^{1 / 2} \sin \xi\right]^{2} / 4 s^{2}\right\} \\
& \times \exp \left\{-s^{2}\left[l\left(1-h^{2}\right)^{-1 / 2}+k y_{0}\right]^{2} / F\right\} \\
B_{l}(h)= & 0,
\end{aligned}
$$

where the beam confinement parameter $s$ is defined by

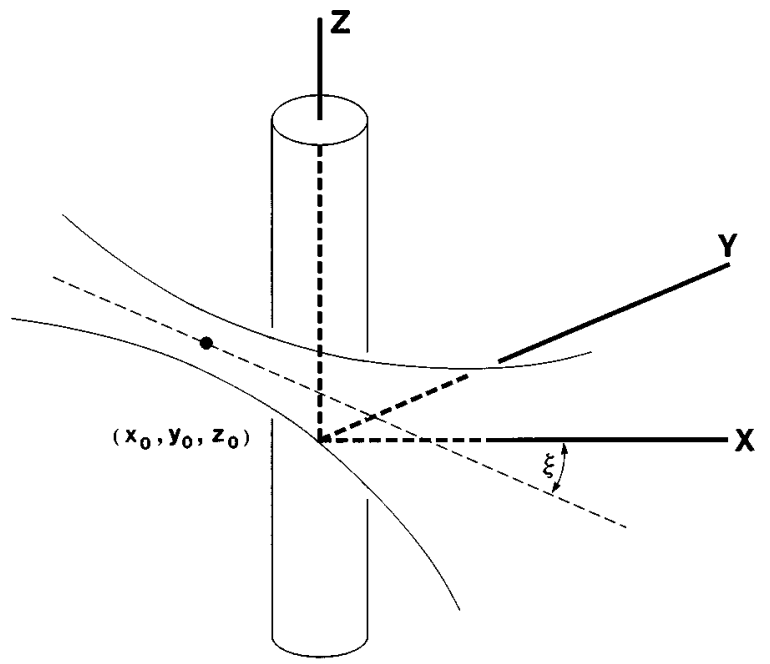

Fig. 1. Geometry of the cylinder and the incident beam. The dominant propagation direction of the incident beam is in the $x-z$ plane and makes an angle $\xi$ with the $x$ axis, and the center of its focal waist is at $\left(x_{0}, y_{0}, z_{0}\right)$.

$$
\begin{aligned}
s \equiv & \frac{1}{k w_{0}}, \\
F= & (\cos \xi)\left[\cos \xi-h\left(1-h^{2}\right)^{-1 / 2}\right. \\
& \times \sin \xi]-2 i s^{2} k x_{0}\left(1-h^{2}\right)^{-1 / 2} .
\end{aligned}
$$

The beam shape coefficients for a $\mu$-polarized incident beam are identical to Eqs. (1)-(3), except that the expressions for $A_{l}(h)$ and $B_{l}(h)$ are interchanged. The variable $h$ in Eqs. (1) and (3) is interpreted as the sine of the angle that a component plane wave in the Fourier angular spectrum of the incident beam makes with the $x$ axis.

The partial-wave amplitudes $\alpha_{l}(h)$ and $\beta_{l}(h)$ of the $\epsilon$-polarized and $\mu$-polarized scattered waves are

$$
\begin{aligned}
& \alpha_{l}(h)=a_{l}(h) A_{l}(h)+q_{l}(h) B_{l}(h), \\
& \beta_{l}(h)=-q_{l}(h) A_{l}(h)+b_{l}(h) B_{l}(h),
\end{aligned}
$$

respectively, where

$$
\begin{aligned}
& a_{l}(h)=\frac{U_{2} W_{1}-n U_{3} W_{3}}{W_{1} W_{2}-n W_{3}^{2}}, \\
& b_{l}(h)=\frac{U_{1} W_{2}-n U_{3} W_{3}}{W_{1} W_{2}-n W_{3}^{2}}, \\
& q_{l}(h)=\frac{2 n l h\left(y^{2}-x^{2}\right)}{\pi x^{2} y^{2}} \frac{J_{l}^{2}(y)}{W_{1} W_{2}-n W_{3}^{2}} .
\end{aligned}
$$

In Eqs. (5) we have

$$
\begin{aligned}
& x \equiv k a\left(1-h^{2}\right)^{1 / 2}, \quad y \equiv n k a\left(1-\frac{h^{2}}{n^{2}}\right)^{1 / 2}, \\
& U_{1}=\frac{n^{2} x}{y} J_{l}(x) J_{l}^{\prime}(y)-J_{l}^{\prime}(x) J_{l}(y), \\
& U_{2}=\frac{n x}{y} J_{l}(x) J_{l}^{\prime}(y)-n J_{l}^{\prime}(x) J_{l}(y), \\
& U_{3}=\frac{h l\left(y^{2}-x^{2}\right)}{x y^{2}} J_{l}(x) J_{l}(y), \\
& W_{1}=\frac{n^{2} x}{y} H_{l}^{(1)}(x) J_{l}^{\prime}(y)-H_{l}^{(1)^{\prime}}(x) J_{l}(y), \\
& W_{2}=\frac{n x}{y} H_{l}^{(1)}(x) J_{l}^{\prime}(y)-n H_{l}^{(1)^{\prime}}(x) J_{l}(y), \\
& W_{3}=\frac{h l\left(y^{2}-x^{2}\right)}{x y^{2}} H_{l}^{(1)}(x) J_{l}(y),
\end{aligned}
$$

where $J_{l}$ are Bessel functions and $H_{l}^{(1)}$ are Hankel functions of the first kind. As is the case for Gaussian-beamsphere scattering, Eqs. (4) are a product of the partialwave scattering amplitudes for plane-wave-cylinder scattering and partial-wave amplitudes of the incident beam. The $a_{l}(h)$ and $b_{l}(h)$ plane-wave amplitudes describe polarization-preserving scattering in the $\epsilon$ and $\mu$ channels, respectively, and the $q_{l}(h)$ plane-wave amplitude describes the cross-polarized scattering $\epsilon$ to $\mu$ and $\mu$ to $\epsilon$.

The $\epsilon$-polarized and $\mu$-polarized partial-wave interior amplitudes are 


$$
\begin{aligned}
& \gamma_{l}(h)=c_{l}(h) A_{l}(h)+p_{l}(h) B_{l}(h), \\
& \delta_{l}(h)=-n p_{l}(h) A_{l}(h)+d_{l}(h) B_{l}(h),
\end{aligned}
$$

respectively, where

$$
\begin{aligned}
& c_{l}(h)=\frac{-2 i n x}{\pi y^{2}} \frac{W_{1}}{W_{1} W_{2}-n W_{3}^{2}}, \\
& d_{l}(h)=\frac{-2 i n x}{\pi y^{2}} \frac{W_{2}}{W_{1} W_{2}-n W_{3}^{2}}, \\
& p_{l}(h)=\frac{-2 n x}{\pi y^{2}} \frac{W_{3}}{W_{1} W_{2}-n W_{3}^{2}} .
\end{aligned}
$$

The interior electric field is

$$
\begin{aligned}
& E_{\text {interior }}(r, \theta, z) \\
& =\frac{E_{0}}{\cos \xi} \int_{-\infty}^{\infty} \mathrm{d} h \sum_{l=-\infty}^{\infty} i^{l+1} \exp (i k h z) \\
& \quad \times \exp (i l \theta)\left\{\left[\frac{i l}{k r} \gamma_{l}(h) J_{l}(\bar{y})+h\left(1-\frac{h^{2}}{n^{2}}\right)^{1 / 2}\right.\right. \\
& \left.\quad \times \delta_{l}(h) J_{l}^{\prime}(\bar{y})\right] \hat{u}_{r}-\left[n\left(1-\frac{h^{2}}{n^{2}}\right)^{1 / 2}\right. \\
& \left.\quad \times \gamma_{l}(h) J_{l}^{\prime}(\bar{y})-\frac{i h l}{n k r} \delta_{l}(h) J_{l}(\bar{y})\right] \hat{u}_{\theta} \\
& \left.-\left[i n\left(1-\frac{h^{2}}{n^{2}}\right) \delta_{l}(h) J_{l}(\bar{y})\right] \hat{u}_{z}\right\},
\end{aligned}
$$

where

$$
\bar{y}=n k r\left(1-h^{2} / n^{2}\right)^{1 / 2} .
$$

Unlike the scattered fields in the far zone, the $h$ integral in Eq. (10) cannot be evaluated by using the method of stationary phase.

\section{S MATRIX}

In atomic, nuclear, and particle physics one is often interested in scattering situations in which a number of different reactions can occur; i.e., for a projectile-target system in a given initial state, many final scattered states are possible. In quantum-mechanical scattering the probability that one of the possible final states will occur in a scattering experiment is given by the magnitude squared of the respective element of the collision matrix, or $S$ matrix, of the system. ${ }^{6}$ In electromagnetic scattering the magnitude squared of the elements of the $S$ matrix describes the fraction of the incident energy channeled into each of the final states. In this section we derive the $S$ matrix for diagonal-incidence arbitrary beam-cylinder scattering. We do this because (1) electromagnetic cylinder scattering provides a prototype example of a nontrivial two-reaction-channel system (the two channels are the polarization states $\epsilon$ and $\mu$ ) for which the $S$ matrix can be calculated exactly, and (2) the mathematical condition for the excitation of MDR's is intimately related to the phase of the elements of the $S$ matrix.
The calculation of the $S$ matrix proceeds as follows. In the spirit of Eqs. (7) we define

$$
\begin{aligned}
& V_{1}=\frac{n^{2} x}{y} N_{l}(x) J_{l}^{\prime}(y)-N_{l}^{\prime}(x) J_{l}(y), \\
& V_{2}=\frac{n x}{y} N_{l}(x) J_{l}^{\prime}(y)-n N_{l}^{\prime}(x) J_{l}(y), \\
& V_{3}=\frac{h l\left(y^{2}-x^{2}\right)}{x y^{2}} N_{l}(x) J_{l}(y),
\end{aligned}
$$

where $N_{l}$ are Neumann functions. If we let

$$
\begin{aligned}
& R_{1} \equiv U_{1} U_{2}-n U_{3}{ }^{2}, \quad R_{2} \equiv V_{1} V_{2}-n V_{3}{ }^{2}, \\
& L_{1} \equiv U_{1} V_{2}-n U_{3} V_{3}, \quad L_{2} \equiv U_{2} V_{1}-n U_{3} V_{3},
\end{aligned}
$$

it may be shown after much algebra that the plane-wave partial-wave scattering amplitudes of Eqs. (5) may be written as

$$
\begin{aligned}
& a_{l}(h)=(1 / 2)\left\{1+M_{l}(h) \exp \left[-i \phi_{l}^{a}(h)\right]\right\} \\
& b_{l}(h)=(1 / 2)\left\{1+M_{l}(h) \exp \left[-i \phi_{l}^{b}(h)\right]\right\} \\
& q_{l}(h)=(1 / 2)\left\{N_{l}(h) \exp \left[-i \phi_{l}^{q}(h)\right]\right\}
\end{aligned}
$$

where

$$
\begin{aligned}
& M_{l}(h)=\left[\frac{\left(R_{1}+R_{2}\right)^{2}+\left(L_{1}-L_{2}\right)^{2}}{\left(R_{1}-R_{2}\right)^{2}+\left(L_{1}+L_{2}\right)^{2}}\right]^{1 / 2}, \\
& N_{l}(h)=\left[1-M_{l}^{2}(h)\right]^{1 / 2}, \\
& \phi_{l}^{a}(h)=\arctan \left[\frac{2\left(R_{1} L_{1}+R_{2} L_{2}\right)}{\left(R_{1}^{2}-R_{2}^{2}\right)-\left(L_{1}^{2}-L_{2}^{2}\right)}\right], \\
& \phi_{l}^{b}(h)=\arctan \left[\frac{2\left(R_{1} L_{2}+R_{2} L_{1}\right)}{\left(R_{1}^{2}-R_{2}^{2}\right)+\left(L_{1}^{2}-L_{2}^{2}\right)}\right] \\
& \phi_{l}^{q}(h)=\arctan \left(\frac{L_{1}+L_{2}}{R_{1}-R_{2}}\right) .
\end{aligned}
$$

By direct substitution it can be verified that the phases in Eqs. (19)-(21) are related by

$$
\phi_{l}^{a}(h)+\phi_{l}^{b}(h)=2 \phi_{l}^{q}(h) .
$$

The leading factor of $1 / 2$ in Eqs. (14) and (15) represents diffraction ${ }^{7}$ and will be examined more fully in a separate paper. ${ }^{8}$ The terms proportional to $M_{l}(h)$ and $N_{l}(h)$ in Eqs. (14)-(16) represent deflection of the plane wave by the cylinder, i.e., reflection, transmission, and transmission following a number of internal reflections. This will also be examined in detail separately. ${ }^{8}$

Using Eqs. (14)-(16), it is now a straightforward matter to calculate the scattering $S$ matrix for arbitrary beam incidence. ${ }^{6}$ First, let the total scalar radiation potential exterior to the cylinder for each partial wave be written as radially incoming part proportional to $H_{l}^{(2)}(k r(1$ $\left.-h^{2}\right)^{1 / 2}$ ) and a radially outgoing part proportional to $H_{l}^{(1)}\left(k r\left(1-h^{2}\right)^{1 / 2}\right)$. The radially incoming part contains a portion of the incident beam in the initial reaction channel (i.e., polarization state) $i$, and the outgoing part con- 
tains the remainder of the incident beam as well as the scattered waves from all the possible final reaction channels (i.e., polarization states) $j$. Then, if the scalar radiation potential is normalized to a unit radially incoming part, the $S$ matrix of the arbitrary diagonal-incidence beam-cylinder system for each partial wave $l$ is defined by

$$
\psi_{l}^{\text {exterior }, i}=H_{l}^{(2)}\left[\begin{array}{l}
A_{l}^{i}(h) \\
B_{l}^{i}(h)
\end{array}\right]-\sum_{j} S_{i j} H_{l}^{(1)}\left[\begin{array}{l}
A_{l}^{i}(h) \\
B_{l}^{i}(h)
\end{array}\right],
$$

where $A_{l}^{i}(h)$ and $B_{l}^{i}(h)$ are the beam shape coefficients of the $i$ th-channel incident beam. Upon substituting Eqs. (4) and (14)-(16) into Eq. (23), we obtain

$$
\begin{aligned}
{[S] } & =\left[\begin{array}{ll}
S_{\epsilon \epsilon} & S_{\epsilon \mu} \\
S_{\mu \epsilon} & S_{\mu \mu}
\end{array}\right] \\
& =\left[\begin{array}{cc}
M_{l}(h) \exp \left(-i \phi_{l}^{a}(h)\right) & N_{l}(h) \exp \left(-i \phi_{l}^{q}(h)\right) \\
-N_{l}(h) \exp \left(-i \phi_{l}^{q}(h)\right) & M_{l}(h) \exp \left(-i \phi_{l}^{b}(h)\right)
\end{array}\right] .
\end{aligned}
$$

Using Eqs. (18) and (22), it is straightforward to show that $[S]$ is unitary, as is required by energy conservation. $^{9}$

If the incoming beam is a plane wave at normal incidence, Eqs. (14)-(16) reduce to

$$
\begin{aligned}
& a_{l}=(1 / 2)\left[1+\exp \left(-2 i \zeta_{l}^{a}\right)\right], \\
& b_{l}=(1 / 2)\left[1+\exp \left(-2 i \zeta_{l}^{b}\right)\right], \\
& q_{l}=0,
\end{aligned}
$$

where

$$
\zeta_{l}^{a}=\arctan \left(V_{2} / U_{2}\right), \quad \zeta_{l}^{b}=\arctan \left(V_{1} / U_{1}\right) .
$$

In this case the $S$ matrix is diagonal,

$$
[S]=\left[\begin{array}{cc}
\exp \left(-2 i \zeta_{l}^{a}\right) & 0 \\
0 & \exp \left(-2 i \zeta_{l}^{b}\right)
\end{array}\right],
$$

indicating that the reaction channels do not communicate, i.e., there is only one possible outgoing polarization state for each incoming polarization state.

\section{MORPHOLOGY-DEPENDENT RESONANCES FOR PLANE-WAVE-CYLINDER SCATTERING}

A MDR of a partial-wave scattering amplitude occurs when the magnitude squared of the amplitude reaches a relative maximum when considered as a function of the cylinder size parameter $k a$. As a specific example, in this section we consider the $m=4$ radial mode of the $l$ $=50$ partial-wave resonance ${ }^{10}$ of the amplitudes $a_{l}(h)$ and $b_{l}(h)$. The behavior of these MDR's is characteristic of all the other MDR's that I have examined. The partial-wave scattering amplitudes $a_{50}(0)$ and $b_{50}(0)$ for a plane wave normally incident on an infinitely long nonabsorbing homogeneous circular cylinder of refractive index $n=1.53$ are plotted in the complex plane in Fig. 2 as a parametric function of the cylinder size parameter $k a$ for $45.75 \leqslant k a \leqslant 46.50$. The $\mathrm{TM}_{50,4}$ and $\mathrm{TE}_{50,4}$ resonances occur in the scattering amplitudes $b_{50}$ and $a_{50}$ for $k a$ $=46.012$ and $k a=46.299$, respectively. The MDR's cor- respond to $\zeta_{l}^{b}=0^{\circ}$ and $\zeta_{l}^{a}=0^{\circ}$ in the normal-incidence $S$ matrix of Eqs. (26) and occur when the plots of $b_{50}(0)$ and $a_{50}(0)$ in Fig. 2 cross the real axis. The other radial modes of the $l=50$ resonances occur when preceding and subsequent cycles of the plots of $b_{l}(0)$ and $a_{l}(0)$ in the complex plane cross the real axis. This zero-phase condition for the normal-incidence $S$ matrix provides a practical way to search numerically for the value of the cylinder size parameter at resonance.

For a diagonally incident plane wave with $h=-\sin \xi$ the situation is more complicated. For example, Fig. 3 shows $a_{l}(-\sin \xi)$ and $b_{l}(-\sin \xi)$ for $l=50$ and $\xi=10^{\circ}$ plotted parametrically in the complex plane as a function of $k a \cos \xi$ for $n=1.53$ and $45.50 \leqslant k a \cos \xi \leqslant 46.25$. Each plot consists of a large circular arc, similar to that for normal incidence in Fig. 2, accompanied by a much smaller circular loop. The radius of the large circular arc is less than $1 / 2$, indicating that only a portion of the incident power is channeled into polarization-preserving scattering. The remaining portion is channeled into the cross-polarized outgoing fields. A similar situation occurs in Fig. 4.1 of Ref. 11. There, the remaining portion

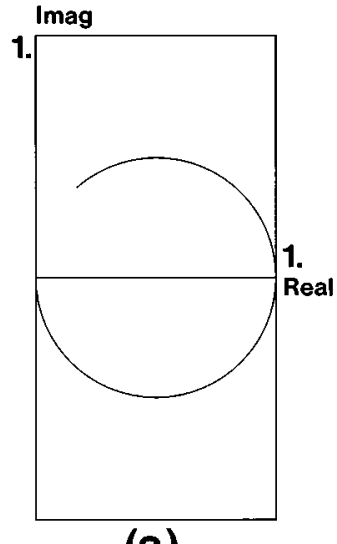

(a)

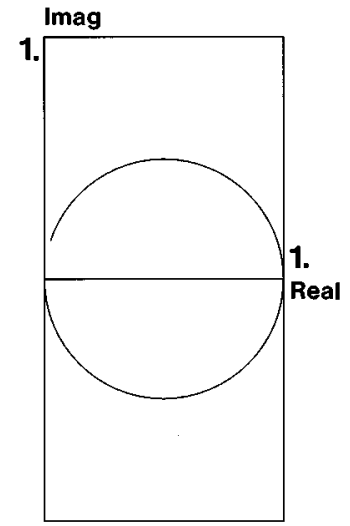

(b)
Fig. 2. Partial-wave scattering amplitudes (a) $a_{l}(-\sin \xi)$ and (b) $b_{l}(-\sin \xi)$ in the complex plane for $l=50, \xi=0^{\circ}$, and 45.75 $\leqslant k a \cos \xi \leqslant 46.50$. The refractive index of the cylinder is $n$ $=1.53$.

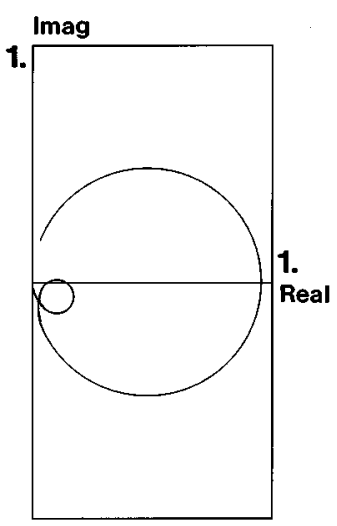

(a)

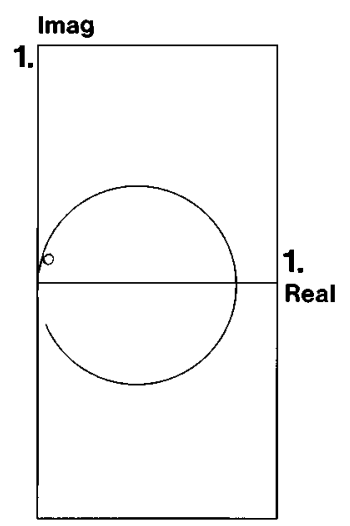

(b)
Fig. 3. Partial-wave scattering amplitudes (a) $a_{l}(-\sin \xi)$ and (b) $b_{l}(-\sin \xi)$ in the complex plane for $l=50, \xi=10^{\circ}, n=1.53$, and $45.50 \leqslant k a \cos \xi \leqslant 46.25$. 
of the incident power is absorbed by the sphere. The location on the large circular arcs that is farthest from the origin corresponds to the size parameter for which the dominant $l=50, m=4$ MDR's occur [i.e., $\mathrm{TM}_{50,4}$ for $b_{l}(h)$ and $\mathrm{TE}_{50,4}$ for $\left.a_{l}(h)\right]$. The location on the much smaller circular loops that is farthest from the origin corresponds to the occurrence of the weaker cross-polarized $l=50, m=4$ MDR's [i.e., $\mathrm{TE}_{50,4}$ for $b_{l}(h)$ and $\mathrm{TM}_{50,4}$ for $\left.a_{l}(h)\right]$.

Our interpretation of Fig. 3 is reinforced in Figs. 4(a), 4(b), and 4(c), where $\left|a_{l}(-\sin \xi)\right|^{2},\left|b_{l}(-\sin \xi)\right|^{2}$, and $\left|q_{l}(-\sin \xi)\right|^{2}$, respectively, are plotted as a function of $k a \cos \xi$ for $l=50, \xi=10^{\circ}, n=1.53$, and 45.50 $\leqslant k a \cos \xi \leqslant 46.25$. In Fig. 4(b) the dominant $\mathrm{TM}_{50,4}$ resonance of $b_{l}$ occurs at $k a \cos 10^{\circ}=45.593$, or $k a$ $=46.296$. In Fig. 4(a) the dominant $\mathrm{TE}_{50,4}$ resonance of $a_{l}$ occurs at $k a \cos 10^{\circ}=45.955$, or $k a=46.664$. A comparison of these values with the normal-incidence values given in the previous paragraph illustrates that the resonant size parameters of the cylinder increase as the angle of incidence of the plane wave increases.

Figures 4(a)-4(c) illustrate another interesting property of cylinder MDR's. For $\xi \neq 0^{\circ}$ the partial-wave scattering amplitude $b_{l}$ that describes $\mu$-polarizationpreserving scattering and that is associated with $\mathrm{TM}_{l, m}$ resonances at normal incidence also weakly contains the $\mathrm{TE}_{l, m}$ resonances associated with the partial-wave scattering amplitude $a_{l}$ at normal incidence. Similarly, the partial-wave scattering amplitude $a_{l}$ weakly contains the $\mathrm{TM}_{l, m}$ resonances as well as the dominant $\mathrm{TE}_{l, m}$ $\epsilon$-polarization-preserving resonances that occur at normal incidence. The reason why both TE and TM resonances occur in each of the scattering amplitudes for diagonal incidence is as follows. Consider, for example, a $\mu$-polarized plane wave diagonally incident on a cylinder. Upon transmission or at an internal reflection it either remains $\mu$ polarized or becomes $\epsilon$ polarized. If crosspolarized transmission or internal reflection takes place when the cylinder size parameter corresponds to the $\mathrm{TE}_{l, m}$ resonance, the now $\epsilon$-polarized interior wave resonates. If, at a later internal reflection or at its exit from the cylinder, the MDR wave converts back to its original polarization state, the scattered field is $\mu$ polarized and is described by the partial-wave scattering amplitude $b_{l}$. But the $b_{l}$ amplitude carries the imprint of the $\mathrm{TE}_{l, m}$ resonance. This mechanism is illustrated in Fig. 5 and will be justified mathematically through the Debye series expansion of the scattering amplitudes in a separate paper. ${ }^{8}$ The cross-polarized scattering amplitude $q_{l}$ of Fig. 4(c) also exhibits both the $\mathrm{TE}_{50,4}$ and $\mathrm{TM}_{50,4}$ resonances.

In order to quantify the dependence of the resonant size parameters on the angle of incidence of the plane wave, the locations of the $\mathrm{TM}_{50,4}$ and $\mathrm{TE}_{50,4}$ resonances were computed for $0^{\circ} \leqslant \xi \leqslant 30^{\circ}$. The results are shown in Tables 1 and 2, respectively, where the columns labeled $b_{50}, a_{50}$, and $q_{50}$ denote the size parameter of the $\mathrm{TM}_{50,4}$ resonance in each of the three partial-wave scattering amplitudes and the columns labeled $\left|b_{50}\right|_{\max }^{2},\left|a_{50}\right|_{\max }^{2}$, and $\left|q_{50}\right|_{\max }^{2}$ denote the maximum value of the magnitude squared of the scattering amplitudes at resonance. The angle of incidence of the plane wave on the cylinder is $\xi$.
Examination of the tables shows that as $\xi$ increases, the polarization-preserving resonances become weaker, the cross-polarized resonances become stronger, and the

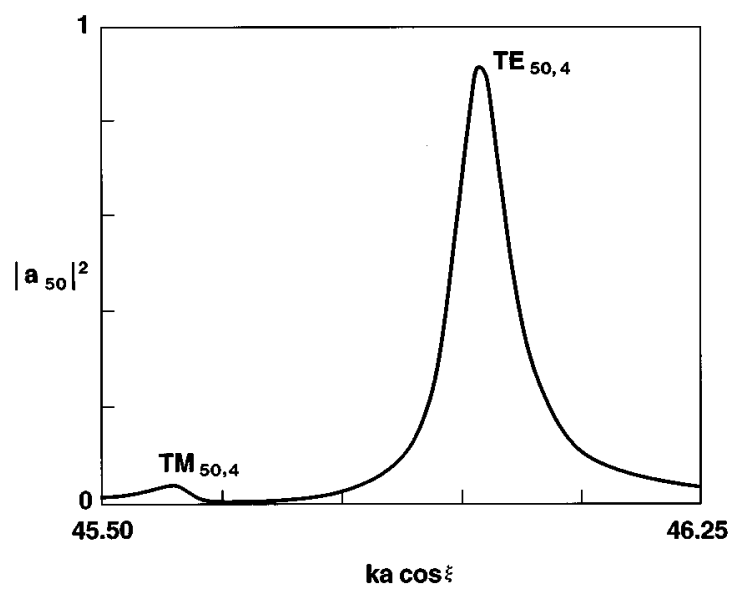

(a)

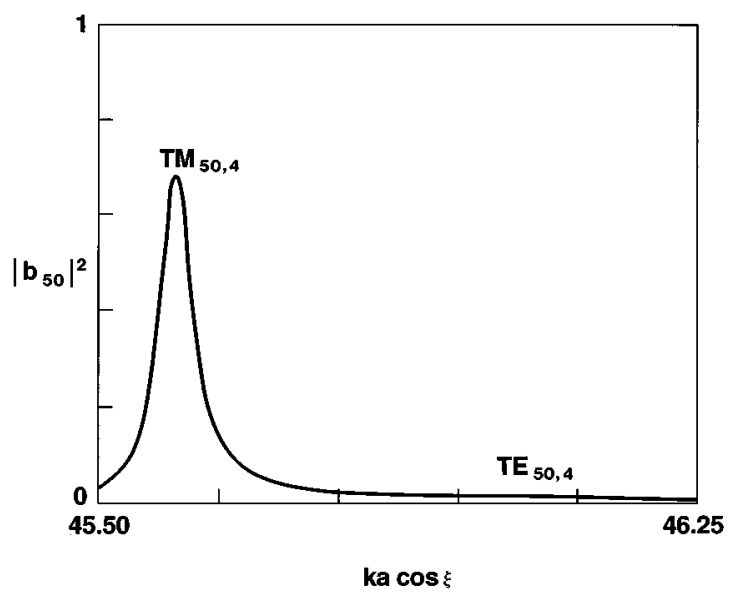

(b)

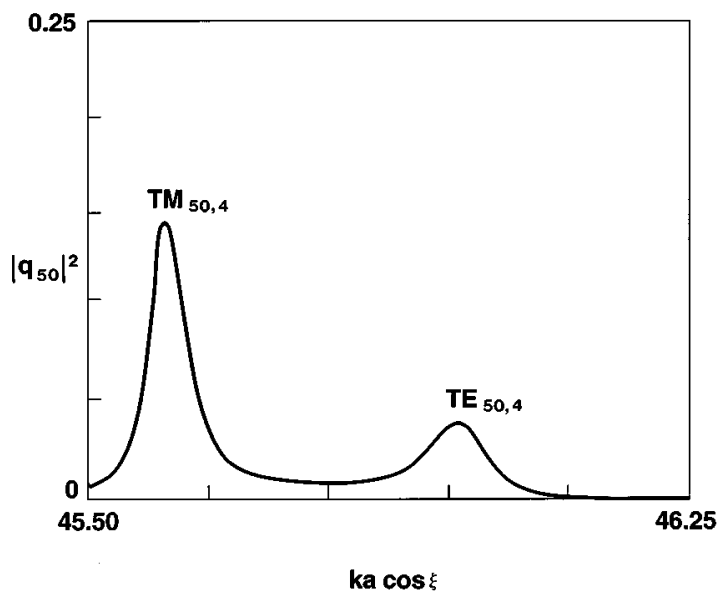

(c)

Fig. 4. Magnitude squared of the partial-wave scattering amplitudes (a) $\left|a_{l}(-\sin \xi)\right|^{2}$, (b) $\left|b_{l}(-\sin \xi)\right|^{2}$, and (c) $\left|q_{l}(-\sin \xi)\right|^{2}$ as a function of $k a \cos \xi$ for $l=50, \xi=10^{\circ}$, and $n=1.53$. The $\mathrm{TE}_{50,4}$ and $\mathrm{TM}_{50,4}$ resonances occur in each scattering amplitude. The $\mathrm{TE}_{50,4}$ resonance in (b) is sufficiently weak that it is not visually apparent. 

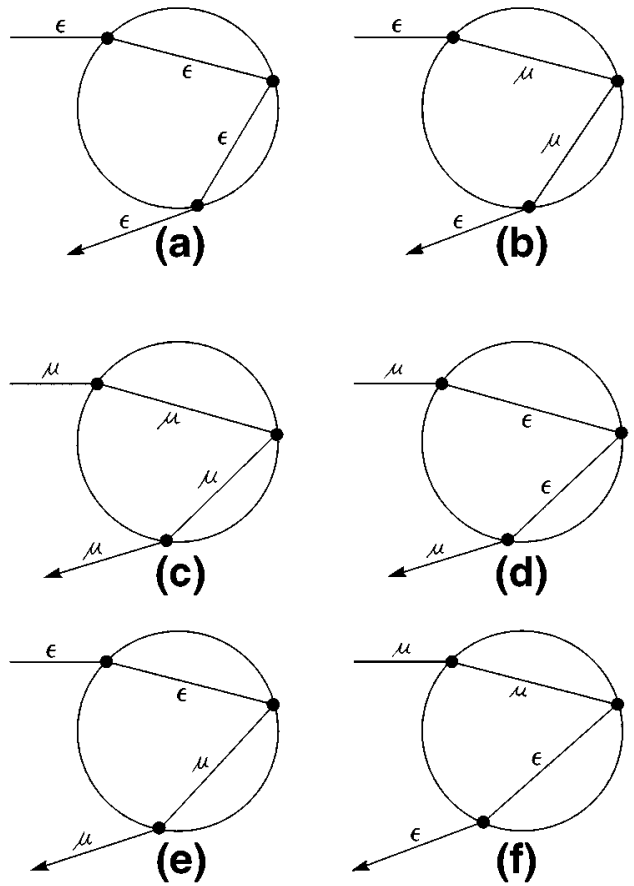

Fig. 5. (a), (b) Two physical processes contributing to $a_{l}(h)$ The process in (a) is polarization preserving, and that in (b) mixes polarizations during the scattering process. (c), (d) Two physical processes contributing to $b_{l}(h)$. The process in (c) is polarization preserving, and that in (d) mixes polarizations during the scattering process. (e), (f) Two physical processes contributing to $q_{l}(h)$.

Table 1. Cylinder Size Parameter $k a$ for the TM $_{50,4}$ Resonance

\begin{tabular}{rcccccc}
\hline$\xi$ & $b_{50}$ & $\left|b_{50}\right|_{\max }^{2}$ & $a_{50}$ & $\left|a_{50}\right|_{\max }^{2}$ & $q_{50}$ & $\left|q_{50}\right|_{\max }^{2}$ \\
\hline $0^{\circ}$ & 46.012 & 1.000 & - & - & - & - \\
$2^{\circ}$ & 46.024 & 0.977 & - & - & 46.025 & 0.011 \\
$4^{\circ}$ & 46.057 & 0.917 & 46.043 & 0.021 & 46.058 & 0.041 \\
$6^{\circ}$ & 46.113 & 0.837 & 46.098 & 0.024 & 46.114 & 0.078 \\
$8^{\circ}$ & 46.193 & 0.753 & 46.181 & 0.031 & 46.194 & 0.115 \\
$10^{\circ}$ & 46.296 & 0.677 & 46.288 & 0.040 & 46.297 & 0.146 \\
$15^{\circ}$ & 46.661 & 0.537 & 46.658 & 0.074 & 46.661 & 0.196 \\
$20^{\circ}$ & 47.179 & 0.454 & 47.179 & 0.107 & 47.179 & 0.220 \\
$25^{\circ}$ & 47.850 & 0.405 & 47.850 & 0.133 & 47.850 & 0.231 \\
$30^{\circ}$ & 48.666 & 0.371 & 48.666 & 0.153 & 48.666 & 0.238 \\
\hline
\end{tabular}

Table 2. Cylinder Size Parameter $k a$ for the $\mathbf{T E}_{50,4}$ Resonance

\begin{tabular}{rcccccc}
\hline$\xi$ & $a_{50}$ & $\left|a_{50}\right|_{\max }^{2}$ & $b_{50}$ & $\left|b_{50}\right|_{\max }^{2}$ & $q_{50}$ & $\left|q_{50}\right|_{\max }^{2}$ \\
\hline $0^{\circ}$ & 46.299 & 1.000 & - & - & - & - \\
$2^{\circ}$ & 46.314 & 0.995 & - & - & 46.295 & 0.003 \\
$4^{\circ}$ & 46.358 & 0.983 & - & - & 46.342 & 0.009 \\
$6^{\circ}$ & 46.432 & 0.964 & - & - & 46.418 & 0.019 \\
$8^{\circ}$ & 46.534 & 0.942 & 46.554 & 0.019 & 46.523 & 0.030 \\
$10^{\circ}$ & 46.664 & 0.919 & 46.684 & 0.017 & 46.655 & 0.041 \\
$15^{\circ}$ & 47.108 & 0.869 & 47.120 & 0.012 & 47.105 & 0.064 \\
$20^{\circ}$ & 47.716 & 0.833 & 47.720 & 0.010 & 47.715 & 0.080 \\
$25^{\circ}$ & 48.480 & 0.809 & 48.480 & 0.011 & 48.479 & 0.091 \\
$30^{\circ}$ & 49.389 & 0.791 & 49.389 & 0.012 & 49.389 & 0.098 \\
\hline
\end{tabular}

widths of the resonances decrease. The resonant size parameter of the dominant $\mathrm{TM}_{50,4}$ resonance of $b_{l}(-\sin \xi)$ is given in the second column of Table 1 and is well fit by the empirical relation

$$
(k a)_{50,4, \mathrm{TM}}^{\mathrm{resonant}}(\xi)=(k a)_{50,4, \mathrm{TM}}^{\mathrm{resonant}}(0)+9.32 \xi^{2},
$$

where $\xi$ is measured in radians. The resonant size parameter of the dominant $\mathrm{TE}_{50,4}$ resonance of $a_{l}(-\sin \xi)$ is given in the second column of Table 2 and is well fit by

$$
(k a)_{50,4, \mathrm{TE}}^{\text {resonant }}(\xi)=(k a)_{50,4, \mathrm{TE}}^{\text {resonant }}(0)+12.02 \xi^{2} .
$$

For $\xi>30^{\circ}$ the resonant size parameters drift above the prediction of Eq. (28) and below that of Eq. (29), indicating the need for higher-order correction terms in these equations. For small $\xi$ the size parameters of the weaker cross-polarized resonances in $a_{l}$ and $b_{l}$, and the resonances in the cross-polarized scattering amplitude $q_{l}$, do not coincide with the size parameters of the dominant resonances. But the differences decrease as $\xi$ increases.

The empirical relations of Eqs. (28) and (29) can be qualitatively justified by using the heuristic model of MDR's. Consider a ray with grazing normal incidence on the cylinder as in Fig. 6(a). At the time $t=0$ a particular location on the incident ray enters the cylinder at point A, becomes an interior MDR wave, and circumnavigates the cylinder just inside its surface by means of successive total internal reflections. At $t=2 \pi a n / c$ it returns to point $\mathrm{A}$ just as the upstream location $\mathrm{C}$ on the incident ray enters the cylinder. Thereafter, the MDR waves from the two locations on the incident ray circumnavigate the cylinder together. If the two locations on

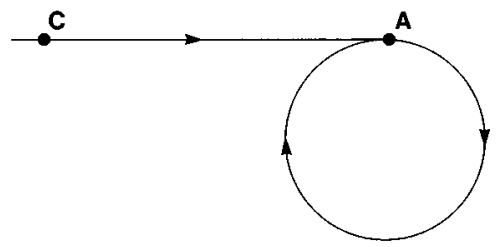

(a)

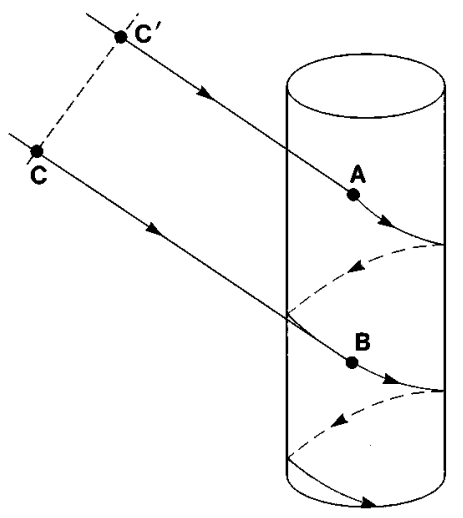

(b)

Fig. 6. (a) Ray with grazing normal incidence on a circular cylinder. The ray enters the cylinder at $A$ and becomes a MDR wave. When it has circumnavigated the cylinder one time, the ray location $\mathrm{C}$ centers the cylinder. (b) Two rays with grazing diagonal incidence on a circular cylinder. When the MDR wave has spiraled down the cylinder one time from point A to point B, the location $C$ on the second ray enters the cylinder at point $B$. 
the incident ray are separated by the distance $l \lambda$, their interior MDR waves are in phase and constructively interfere. This occurs for

$$
l \lambda=c\left(\frac{2 \pi a n}{c}\right)
$$

or

$$
k a=\frac{l}{n} .
$$

Equation (31) is recognized as the zero-order approximation to the resonant size parameter of the $m=1$ radial mode MDR of the partial wave $l$.

Extending this argument to diagonal incidence in Fig. 6(b), we now consider an incident grazing ray that makes an angle $\xi$ with the horizontal plane. At $t=0$ a particular location on the incident ray refracts into the cylinder at point $\mathrm{A}$ and becomes an interior MDR wave. The angle that the refracted wave inside the cylinder makes with the horizontal plane is $\sigma$, where

$$
\sin \xi=n \sin \sigma
$$

by Snell's law. The MDR wave then spirals down the cylinder just inside its surface by means of successive internal reflections. At the time $t=2 \pi a n /(c \cos \sigma)$ it has cycled around the cylinder once and is at point B a distance $z=2 \pi a$ tan $\sigma$ directly below point A. At this time the location $\mathrm{C}$ on a second grazing-incidence ray refracts into the cylinder at point $\mathrm{B}$ and becomes an interior MDR wave. The two MDR waves thereafter spiral down the cylinder together. If the distance $\mathrm{C}^{\prime} \mathrm{A}$ is $l \lambda$, the continued spiraling is in phase and produces constructive interference. This occurs when

$$
l \lambda+2 \pi a \tan \sigma \sin \xi=c\left(\frac{2 \pi a n}{c \cos \sigma}\right),
$$

or

$$
k a \approx \frac{l}{n}+\xi^{2}\left(\frac{l}{2 n^{3}}\right)
$$

for small $\xi$. The quadratic dependence of relation (34) on $\xi$ agrees with the empirical relations of Eqs. (28) and (29). For $l=50$ and $n=1.53$ we have $l / 2 n^{3} \approx 6.98$, which is within a factor of 2 of the coefficient of $\xi^{2}$ in Eqs. (28) and (29) for the $l=50, m=4$ resonances. For the $l=50$, $m=1$ resonances, which we also numerically computed, the empirically obtained coefficients of the resonant size parameter equations analogous to Eqs. (28) and (29) are 7.54 and 8.46 , respectively. These values are only $8 \%$ and $12 \%$ higher than the prediction of relation (34), thus lending credence to the mechanism of Fig. 6(b).

For normal plane-wave incidence the $\mathrm{TM}_{l, m}$ and $\mathrm{TE}_{l, m}$ resonances occur when the phases $\zeta_{l}^{b}$ and $\zeta_{l}^{a}$, respectively, pass through $0^{\circ}$. At the dominant $\mathrm{TM}_{50,4}$ and $\mathrm{TE}_{50,4}$ resonances for diagonal plane-wave incidence the phases $\phi_{l}^{b}$ and $\phi_{l}^{a}$ of Eqs. (20) and (19), respectively, do not pass through $0^{\circ}$ at the exact locations of the maxima of $\left|b_{l}\right|^{2}$ and $\left|a_{l}\right|^{2}$. But, for $0^{\circ} \leqslant \xi \leqslant 30^{\circ}$, the largest size parameter difference between the $\phi_{l}^{b}=0^{\circ}$ condition and the relative maximum condition for the dominant $\mathrm{TM}_{50,4}$ resonance in $b_{l}(h)$ is less than $\Delta k a=0.0002$, and the largest difference for the dominant $\mathrm{TE}_{50,4}$ resonance in $a_{l}(h)$ is less than $\Delta k a=0.0007$. Thus searching for the zeros of $\phi_{l}^{b}$ and $\phi_{l}^{a}$ in the $S$ matrix of Eq. (24) again provides a practical method for approximating the resonance size parameters for diagonal-incidence-cylinder scattering.

As is the case for normal incidence, greatly enhanced interior fields are associated with a MDR produced by a diagonally incident plane wave. The resonant behavior of $c_{l}(h), d_{l}(h)$, and $p_{l}(h)$ parallels that of $a_{l}(h), b_{l}(h)$, and $q_{l}(h)$, respectively. The interior amplitude $c_{l}(h)$ produces a strong $\mathrm{TE}_{50,4}$ resonance and a weaker $\mathrm{TM}_{50,4}$ resonance. Similarly, $d_{l}(h)$ possesses a strong $\mathrm{TM}_{50,4}$ resonance and a weaker $\mathrm{TE}_{50,4}$ resonance. As $\xi$ increases, the peak value of the interior fields increases, which is expected, since the resonances are becoming sharper. The cross-polarized interior amplitude $p_{l}(h)$ also possesses both resonances. The peak interior field strengths do not occur exactly at the same values of $k a$ as do the maximum values of the partial-wave scattering amplitudes, because of the size parameter dependence of $W_{1}, W_{2}$, and $W_{3}$ in Eq. (7). But the largest differences were found to be $\Delta k a=0.0005$ for the $\mathrm{TM}_{50,4}$ and $\mathrm{TE}_{50,4}$ dominant resonances in $d_{l}(h)$ and $c_{l}(h)$, respectively.

\section{NOVEL FEATURES OF MORPHOLOGY-DEPENDENT RESONANCES FOR GAUSSIAN-BEAM-CYLINDER SCATTERING}

For light scattering by a sphere the size parameters of the MDR's are independent of whether the incident beam is a plane wave, a Gaussian beam, or some other beam with a more complicated profile. This is because (1) the partialwave scattering amplitudes are a product of the planewave scattering amplitudes and the spherical coordinate beam shape coefficients, and (2) resonant structure occurs only in the plane-wave scattering amplitudes. For cylinder scattering we saw in Eqs. (4) that the partial-wave scattering amplitudes are also the product of the planewave scattering amplitudes and the cylindrical coordinate beam shape coefficients. But in this case there is an influence of the shape of the beam on the MDR that is not found in sphere scattering, as a result of the incident beam being represented by an angular spectrum of plane waves having different values of $h$ corresponding to different angles of incidence.

One predicted difference between MDR's produced by plane-wave incidence and Gaussian-beam incidence is qualitatively illustrated in Figs. 7 and 9. In Fig. 7(a) a $\mu$-polarized plane wave is normally incident on a cylinder whose size parameter corresponds to a TM resonance. As a result, the scattered intensity is resonantly enhanced along the entire length of the cylinder. Similarly, in Fig. 7(b) the plane wave is normally incident on a cylinder whose size parameter is slightly larger than that for the TM resonance. The resulting scattering is nonresonant. In Paper I we saw that far-zone scattering of a Gaussian beam in each direction $\eta$ as in Fig. 8 corresponds to scattering by a different diagonally incident component plane wave in the Fourier angular spectrum of the incident beam. This has interesting implications for 

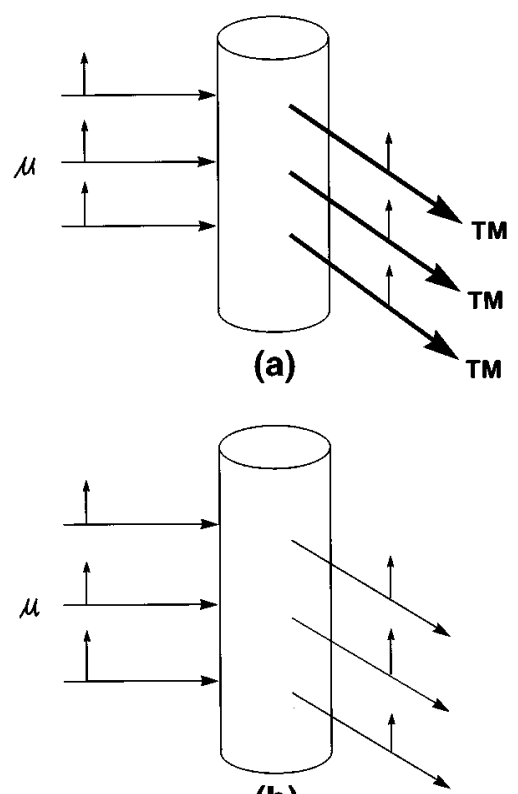

(b)

Fig. 7. A $\mu$-polarized incident plane wave produces (a) resonant scattering if the cylinder size parameter corresponds to TM resonance and (b) nonresonant scattering if the cylinder size parameter is slightly larger than that for a TM resonance.

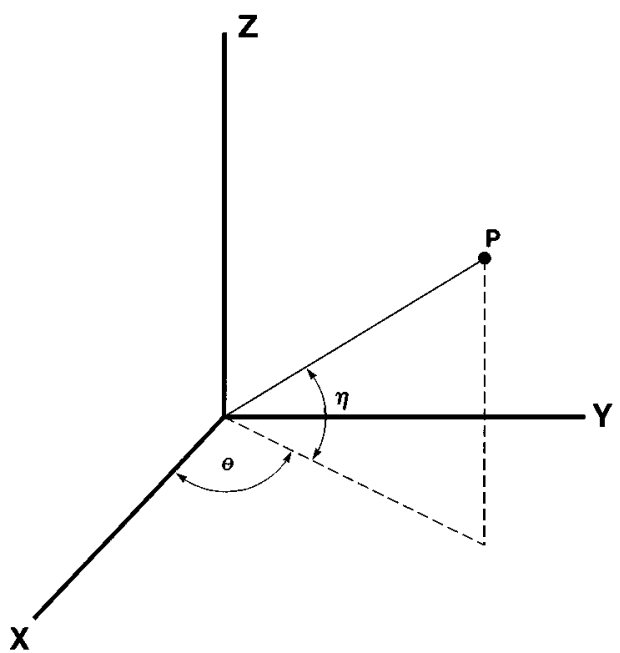

Fig. 8. Geometry of the far zone. The coordinates of the point $P$ in the far zone are $(x, y, z)$. The line joining $P$ to the origin makes an angle $\eta$ with the horizontal plane, and its projection onto the horizontal plane makes an angle $\theta$ with the $x$ axis.

MDR production. Consider, for example, the situation in Fig. 9(a), where an on-axis $\mu$-polarized Gaussian beam is normally incident on a cylinder having the size parameter for which a normally incident plane-wave TM resonance would occur. Equation (28) predicts that scattering of the Gaussian beam in the $\eta=0^{\circ}$ horizontal plane is resonant, while scattering both above and below the horizontal plane is nonresonant because the size parameter for diagonal-incidence resonance is increased. In Fig. 9(b) the Gaussian beam is normally incident on a cylinder having the size parameter slightly larger than that for which a normally incident plane-wave TM resonance would occur. Scattering in the horizontal plane is now nonresonant, while scattering at some angle both above and below the horizontal plane is resonant. Finally, in Fig. 9(c) the $\mu$-polarized on-axis Gaussian beam is normally incident on a cylinder having the size parameter slightly larger than that of an $\epsilon$-polarized TE plane-wave resonance. The $\mu$-polarized scattering in the horizontal plane is nonresonant, while the $\mu$-scattered waves and the $\epsilon$-scattered waves at some angle both above and below the horizontal plane are resonant. The situation is similar for a diagonally incident Gaussian beam.

If an off-axis Gaussian beam, traveling parallel to the $x$ axis as in Fig. 1 and polarized in the $z$ direction, were incident on a dielectric sphere, again both TE and TM resonances would be produced. ${ }^{12}$ But for sphere scattering the reason for this is fundamentally different from that
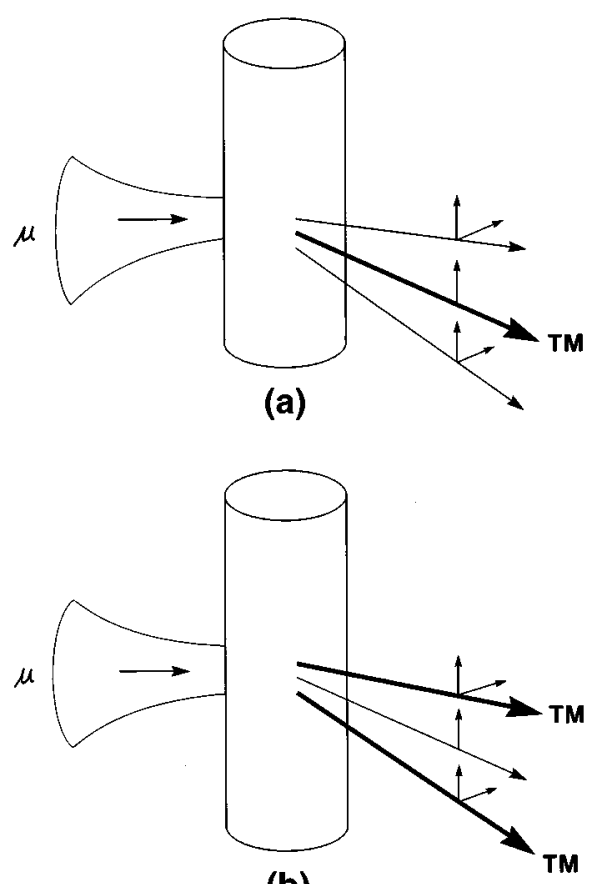

(b)

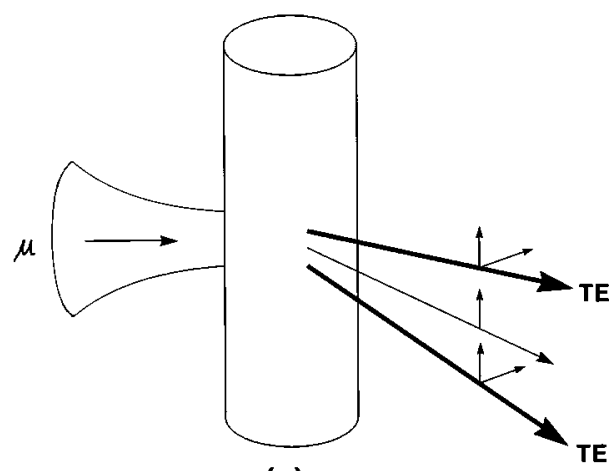

(c)

Fig. 9. A $\mu$-polarized incident Gaussian beam produces (a) resonant scattering in the horizontal plane and nonresonant scattering above and below the horizontal plane if the cylinder size parameter corresponds to a plane-wave TM resonance, (b) nonresonant scattering in the horizontal plane and resonant scattering at some angle above and below the horizontal plane if the cylinder size parameter is slightly larger than that for a plane-wave TM resonance, and (c) nonresonant scattering in the horizontal plane and resonant scattering at some angle above and below the horizontal plane if the cylinder size parameter is slightly larger than that for a plane-wave TE resonance. 
for cylinder scattering. For sphere scattering both TE and TM resonances are produced because the partialwave expansion of an off-axis beam in spherical coordinates possesses both TE beam shape coefficients $B_{l m}$ and TM beam shape coefficients $A_{l m}$. For cylinder scattering both TE and TM resonances are produced because the plane-wave partial-wave scattering amplitudes $b_{l}(h)$ and $a_{l}(h)$ themselves mix the TE and TM resonances by means of the polarization-preserving and cross-polarized transmission and successive internal reflection mechanism of Fig. 5.

Another difference between MDR's produced by planewave incidence and Gaussian-beam incidence concerns the internal fields at resonance. Consider a tightly focused Gaussian beam diagonally incident on a cylinder whose size parameter corresponds to a MDR, and let the beam be positioned at the edge of the cylinder so as to maximize the MDR production rate. One intuitively expects that the interior resonant fields spiral down the cylinder just inside its surface far beyond the localized region of initial illumination because of successive internal reflections, as is illustrated in Fig. 6(b). This has been observed experimentally ${ }^{13}$ and agrees with the heuristic model of MDR's, assuming that we apply it individually to each of the component plane waves in the beam's Fourier angular spectrum.

\section{CONCLUSIONS}

In this paper and in Paper I we considered scattering of a diagonally incident focused Gaussian beam by an infinitely long homogeneous circular cylinder. In Paper I we found that the key to both the solution of the problem and its physical interpretation was parameterizing the incident beam as an angular spectrum of plane waves. In this paper we were interested in the scattering $S$ matrix and its relation to the mathematical condition for the production of MDR's. As was shown in Section 3, diagonalincidence cylinder scattering is a nontrivial example of a physical system having two reaction channels and whose $S$ matrix can be evaluated exactly. In Sections 4 and 5 we saw that TE and TM resonances are coupled in the scattering amplitudes. The nature of the coupling is intrinsic; i.e., it occurs in the plane-wave partial-wave scat- tering amplitudes themselves rather than in a mixing of different polarization incident beam shape coefficients. Because of this, the MDR's for diagonal plane-wavecylinder scattering and for diagonal Gaussian-beamcylinder scattering enjoy a number of properties not shared by sphere MDR's and should lead to an interesting extension of MDR spectroscopy.

\section{REFERENCES}

1. S. C. Hill and R. E. Benner, "Morphology-dependent resonances," in Optical Effects Associated with Small Particles, P. W. Barber and R. K. Chang, eds. (World Scientific, Singapore, 1988), pp. 3-61.

2. J. P. Barton, D. R. Alexander, and S. A. Schaub, "Internal fields of a spherical particle illuminated by a tightly focused laser beam: focal point positioning effects at resonance," J. Appl. Phys. 65, 2900-2906 (1989).

3. G. Chen, R. K. Chang, S. C. Hill, and P. W. Barber, "Frequency splitting of degenerate spherical cavity modes: stimulated Raman scattering spectrum of deformed droplets," Opt. Lett. 16, 1269-1271 (1991).

4. G. Chen, M. M. Mazumder, Y. R. Chemla, A. Serpenguzel, R. K. Chang, and S. C. Hill, "Wavelength variation of laser emission along the entire rim of slightly deformed microdroplets," Opt. Lett. 18, 1993-1995 (1993).

5. J. A. Lock, "Scattering of a diagonally incident focused Gaussian beam by an infinitely long circular cylinder," J. Opt. Soc. Am. A 14, 640-652 (1997).

6. S. de Benedetti, Nuclear Interactions (Wiley, New York, 1964), pp. 322, 326-327.

7. H. C. van de Hulst, Light Scattering by Small Particles (Dover, New York, 1982), pp. 209-210.

8. J. A. Lock and C. L. Adler, "Debye series analysis of the first-order rainbow produced in scattering of a diagonally incident plane wave by a circular cylinder," J. Opt. Soc. Am. A (to be published).

9. Ref. 6, p. 330 .

10. J. F. Owen, P. W. Barber, P. B. Dorain, and R. K. Chang, "Enhancement of fluorescence induced by microstructure resonances of a dielectric fiber," Phys. Rev. Lett. 47, 10751078 (1981).

11. M. Kerker, The Scattering of Light and Other Electromagnetic Radiation (Academic, New York, 1969), p. 98.

12. J. A. Lock, "Improved Gaussian beam scattering algorithm," Appl. Opt. 34, 559-570 (1995).

13. R. G. Pinnick, G. L. Fernandez, J.-G. Xie, T. Ruekgauer, J. $\mathrm{Gu}$, and R. L. Armstrong, "Stimulated Raman scattering and lasing in micrometer-sized cylindrical liquid jets: time and spectral dependence," J. Opt. Soc. Am. B 6, 865870 (1992). 